\title{
Progress with Restoration: Our Widening Scope
}

"A re we making progress?" is a constant, and rather healthy, question for any cognate discipline or activity to ask itself. I am pleased to see that this is a major topic in this Special Issue of Ecological Restoration, as it is a question I often reflect on myself. I have little doubt that in terms of raising ecological restoration's profile in national and international arenas, there have been some outstanding and remarkable successes. Ecological restoration is a key plank of the IUCN/CBD programs to combat the loss of biodiversity, moving the discussion beyond conservation to an active engagement with restoring what we have lost. Ecological restoration is also a key focus of the European Union's EU 2020 target and 2050 biodiversity vision as adopted by the European Council in March 2010, which extends beyond conservation and maintenance of biodiversity to restoration of degraded ecosystems and ecosystem services; these targets need to be taken into the global arena.

There remain some knotty questions to address, however. How we measure the success of restoration has been approached from widely differing angles. Many programs have been aimed at single species or groups of species, or general biodiversity measures-targeting birds, for example, has brought about some startling progress. The areal extent of habitat types has also been a target, often focused on single systems, such as wetlands, heathland, or woodlands. In many of these cases, we have to take great care that by focusing on one set of criteria we do not limit the chances of success in others. Traditional approaches centered on historical ecosystems are not applicable in many parts of the world, especially the heavily industrialized or postindustrial societies, and we must look to other targets and standards.

This brings us to the work of bringing systems approaches to not only monitoring restoration success but actually using system parameters as targets themselves. For example, work has been carried out using ecosystem function, such as minimization of nitrogen losses through the use of buffer strips on the edge of agricultural areas. There are also some more esoteric measurements we might take, such as determination of the thermodynamic efficiency of forests, or $1 / F$ "power law" relationships between amplitude and frequency of occurrences - how big are fires and how often do we have them, for example. These are related to Odum's theories of ecosystem development and maturity, which must have a place in our considerations.

This then lends itself to the whole arena of ecosystem services, which have captured the imagination of policy makers around the world. Importantly, this group includes finance ministers, making the chances of this type of approach being put into action a reality. Carbon sequestration, flood risk minimization, biodiversity, and cultural values are encapsulated in this approach and truly address the agenda of restoring ecosystems. We as a community have to be prepared to interpret and communicate what we are trying to achieve to many different audiences, and knowing how to address different sectors and interests successfully will bring about achievement of the goal of reconnecting nature with culture.

Finally, on a personal note, this is sadly my last editorial as Chair of SER. I'd like to thank the editorial team, Mrill Ingram and Chris Reyes, for their support, correction of my grammar, and translation of my text into U.S. English! We are very fortunate in that Dr. Steve Whisenant of Texas A\&M will be taking up the reins in August; you will be hearing from him next. Thank you for listening!
Jim Harris
Chair
Society for Ecological Restoration 